\title{
A mobile Internet Applications System Based on Cloud Computing and Identification
}

\author{
Yang Li, Jie Li \\ DIGITAL CHINA(CHINA)LIMITED, Beijing, China \\ adigital9898@sina.com
}

\begin{abstract}
A mobile Internet applications system based on identity recognition technology is proposed, which can achieve user identification, analysis and as a basis for realization of intelligent portal system. The system support the development of more flexible operational partners and loading mode based on user behavior cloud computing technology, to provide users with online cloud-virus monitoring system.

Keywords: Mobile internet application, Cloud computing, Identification recognition

\section{Introduction}

With the rapid progress of information society in recent years, mobile Internet field is increase to obtain rapid development in this context.

To full use the advantages of the mobile network, based on cloud computing technology, combined with mobile subscriber identity and behavior analysis, data mining techniques, we can discover the value of the means, while the introduction of SNS concept of operations and other comprehensive mobile Internet applications.

The system can not only provide users with the entertainment, socializing, shopping to the full range of industrial applications, such as mobile Internet experience, but also for the rapid deployment of new services has laid a solid foundation for the development of the mobile Internet field.
\end{abstract}

\section{System architecture design}

The system is based on Cloud computing and identification techniques, to discover the value of data mining and Internet applications, which uses a hierarchical design approach, shown in fig 1 , the whole system is divided into: 


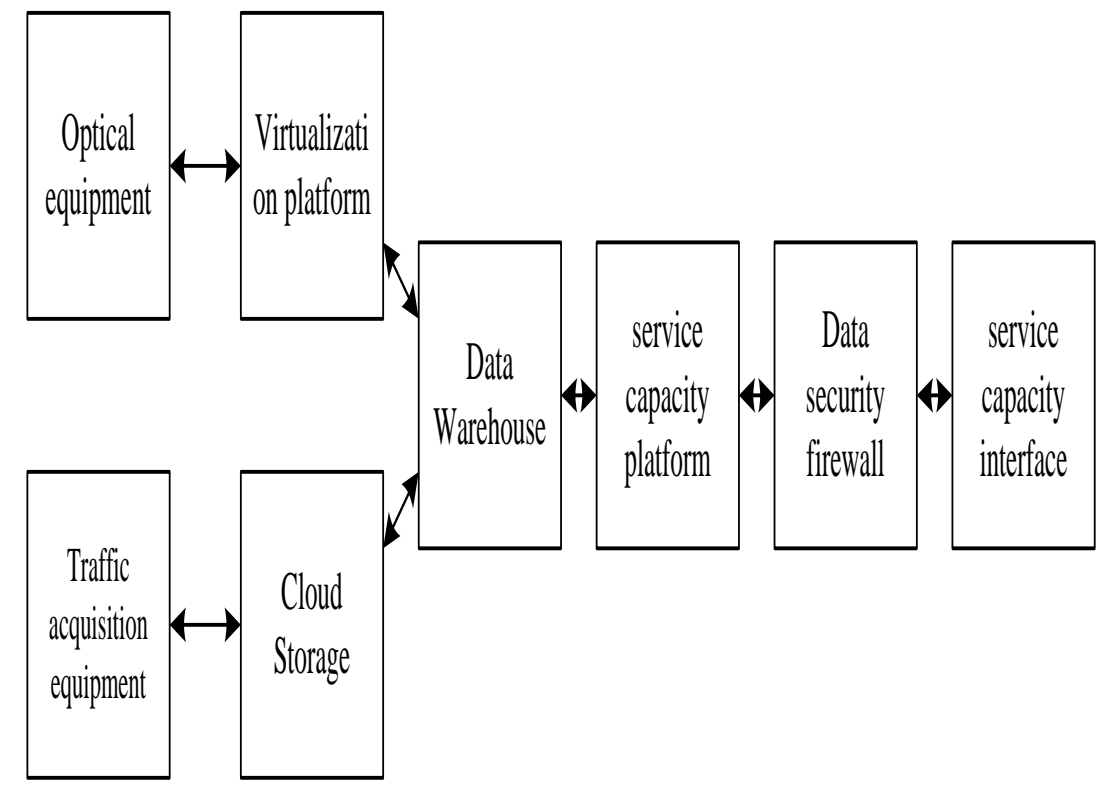

Figure 1. System hierarchy diagram

The whole network user's full traffic capture and DPI.

Cloud capability layer: Based on the underlying hardware virtualization technology, provide the basis for computing and storage resources for data layer and business capability layer capabilities.

Data capacity Layer: The data mining techniques from traffic acquisition layer for large data storage and processing analysis.

Operational capacity Layer: provides services for users or collaborators capabilities, such as identification systems, intelligent gateway system, users access the navigation system, personalized mobile newspaper platform.

Secure Access Layer: to protect users' privacy data for the purpose of providing the public network to the set of interfaces collaborators.

\section{Cloud ability layer architecture}

Cloud capability layer logic architecture is shown in fig 2. 


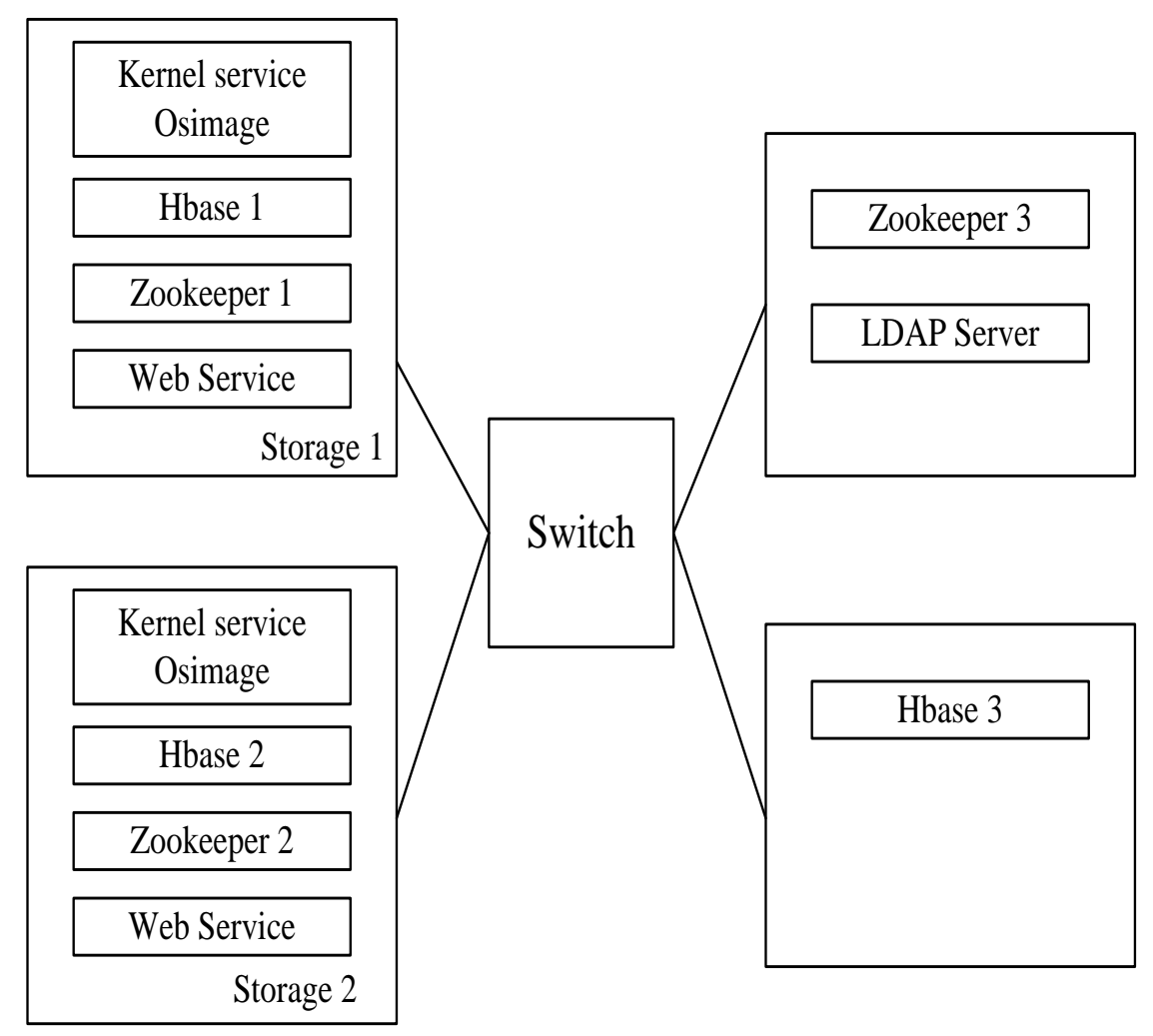

Fig 2. Cloud capability layer logic architecture

Cloud computing resource capacity layer divided into two categories: compute nodes, the storage node. Computing node is responsible for providing a virtual machine's computing power; storage nodes and disk array connected to provide storage resources for virtual machines.

Core components function of the platform is as follows:

\section{1) $\mathrm{PXE}$}

PXE service node provides DHCP, DNS, HTTP and TFTP services. PXE server is used to create a mirror and configure, other computers need to use other machines quickly installed over the network.

\section{2) ZooKeeper}

ZooKeeper is an open source project of Apache Hadoop. It is a scalable distributed lock service. As shown, ZooKeeper must be deployed in the odd number nodes, the number of nodes in the cloud platform in ZooKeeper at least is three.

3) Web Service

In the cloud platform, at least one or more of the Web Service node need to run. Multiple Web Service nodes can be distributed through software or hardware load balancer to achieve REST request load.

4) Web Control 
At least one Web console is needed to provide a Web interface support. As Web Service nodes, multiple Web console can be run simultaneously on multiple nodes, load distribution achieved through the load balancer.

5) LDAP Server

LDAP provides user authentication for Web service.

6) Hbase

Hbase is the Apache Hadoop open source project. HBase is a distributed, scalable, column-oriented data storage based on the Google BigTable. The recommended minimum configuration requires three nodes running HBase services.

\section{Data capacity Layer design}

Through analysis of the basic features of the customer, customer behavior preferences, customer spending power, channel preferences of customers, customer call behavior, customer life trajectories, customer relationship circle, terminal services matching behavior, portraits for clients to form the customer tag library, fully refined customers. Through customer segmentation, customer clustering, establish target customer base, its data analysis results can be used as input refined operational systems, intelligent portals and other systems

\section{Service capacity subsystem design}

The system includes the following operational capacity subsystems:

Subscriber Identity: Get User's MDN number in 3G mode and provides access to the other business platform.

NET billing subsystems: user identity, based on the current network VAC docking system provides collaboration services public network authentication, accounting.

Intelligent Portal: personalized portal showing different content for different users to obtain the user's identity and attribute information such as the user's interest.

The refine operational platforms: through user-depth analysis of Internet traffic, to learn the user attributes and other information of interest to complete the user portrait, thereby providing intensive operations and business support for other platforms.

Internet users navigation system: Push particular Internet address to a specific user based on specific policies, and thus play a role in the promotion of its own portals.

Design goals of the subsystem include, but are not limited to:

Support a unified user interface property acquisition, support data security firewall mechanism.

The system needs to have good security, establish a complete security system and rights management, to ensure business information processing and data transmission correctness and security. 
Use of hierarchical, component design, application software and system isolation underlying functional modules, easy application modification and maintenance of system software, with good portability

Provide a unified external interface based on WEB SERVICE, easy information exchange with other user applications.

To consider the development needs of the system, the need to set aside a certain number of functional interfaces for scalability.

Support $7 \times 24$ uninterrupted operations.

Service processes design

The system and various subsystems follow a unified data access and data distribution processes in order to continue to expand the system and later maintenance.

User initiates a network access request

SP initiate to identify the user's authorization ID to the system

System provide an authorization unique ID matched with the user for SP

$\mathrm{SP}$ add request to authorization identifier, returned to the user

The user initiates an authentication request to the system, Gn interface data acquisition, analysis, build authorization ID, MSISDN, private IP, product ID and other information complete correspondence table

SP queries to obtain user identification number under the authority

Promote the business or complete authentication.

\section{NET billing subsystem}

The processes of net billing subsystem are:

SP purchase request submitted by the user

SP charging gateway to NET application SKEY; (interface 1)

NET billing gateway SP authentication (SPID, SP passwords, product ID, source IP), returns the SP a SKEY4) SP notifies the user browser steering NET billing gateway for billing

User initiates charging request to NET Charging Gateway (SPID, SP code, product name, SKEY, callback url, redirect url, image url); (Interface 2)

NET charging gateway billing confirmation message is returned to the user (including SP names, product images, product name, phone number chargeback, chargeback amount), product images provided by the image url

User clicks "OK" to confirm deductions

NET confirmation soon chargeback billing gateway to the SP through callback url; (Interface 3)

SP confirm deductions

NET VAC charging gateway to make deductions apply

VAC return chargeback results

NET Charging Gateway notify SP accounting results (via callback url); (Interface 3)

SP confirm billing results

NET gateway to notify the user chargeback billing success 
Users to access relevant business

\section{Users navigate subsystem business processes}

The processes of users navigate subsystem business are:

User initiates a PDP activation request

GGSN in response to the request, and returns the IP address assigned to the user 3) the user initiates an HTTP GET request, request access to the site

Navigation platform listening to the Gn interface data, according to the policy judgment for the first PDP request and intercept the connection

Judge the target URL navigation platform, whether it is in white list, whether the user is in the white list

Navigation platform push navigation system requests a page, carry information users MSISDN, UA, target URL, etc.

Navigation system organizations a navigation page, add the user's original access link

Navigation system returns to the navigation page navigation platform

Navigation platform returns navigation page to the user.

\section{Intelligent portal subsystem business processes}

The processes of intelligent portal subsystem business are:

User access to intelligent portal system.

Get Smart portal user identity through user identification system

Intelligent Portal system initiates a query to the mobile phone number refined operating platform

Refine operating platform return user attributes

Intelligent Gateway generates personalized portal pages and content based on user attributes returned.

\section{Conclusion}

This paper proposes a mobile Internet applications and cloud-based identity recognition technology, can achieve user identification, analysis and as a basis for realization of intelligent portal system, portal development in their own ability to achieve the lead, develop and run the engine PaaS mode to achieve a single portal platform based on user behavior cloud computing technology on supporting the development of more flexible operational partners and loading mode, to provide users with online cloud-virus monitoring system, and uses the first "data security firewall" solution.

\section{Acknowledgements}

This work was financially supported by the project (2012AA01A403). 


\section{References}

[1] Bao Rong Chang ; Hsiu Fen Tsai, in: Access Security on Cloud Computing Implemented in Hadoop System, Genetic and Evolutionary Computing (ICGEC), 2011 Fifth International Conference on, 2011

[2] Chenguang Wang ; Huaizhi Yan, in: Study of Cloud Computing Security Based on Private Face Recognition, Computational Intelligence and Software Engineering (CiSE), 2010 International Conference on, 2010

[3] Petcu, D. in: Identifying Cloud computing usage patterns, Cluster Computing Workshops and Posters (CLUSTER WORKSHOPS), 2010

[4] Al Rassan, I. ; AlShaher, H. in : Securing Mobile Cloud Computing Using Biometric Authentication (SMCBA) , Computational Science and Computational Intelligence (CSCI), 2014 International Conference on, 2014

[5] Jucheng Yang ; Naixue Xiong, in: A Fingerprint Recognition Scheme Based on Assembling Invariant Moments for Cloud Computing Communications, 2011 